\title{
DESARROLLO DE OPORTUNIDADES SOCIALES SOSTENIBLES: COMUNIDAD DEL BARRIO EL DORADO
}

\section{DEVELOPMENT OF SUSTAINABLE SOCIAL OPPORTUNITIES: COMMUNITY OF NEIGHBORHOOD EL DORADO}

\author{
Janneth Palacios Chavarro*
}

\section{RESUMEN}

Este artículo presenta los principales resultados del diagnóstico a la comunidad del barrio El Dorado (Bogotá, Colombia), identificando sus necesidades, debilidades, potencialidades, amenazas y oportunidades. Analiza la dinámica de la comunidad y su realidad como fenómeno de transformación endógena y propone fortalecer su comunicación y participación social para iniciar procesos de cambio y de convergencia con las acciones y políticas públicas locales y nacionales.

PALABRAS CLAVE: COLOMBIA * DESARROLLO SOCIAL * COMUNIDADES * PARTICIPACIÓN COMUNITARIA * POLITICA DE DESARROLLO * COMUNICACIÓN

\section{ABSTRACT}

This article presents the main results of the diagnosis realized to the community of neighborhood El Dorado, Bogota (Colombia), identifying its needs, weaknesses, potentials, threats and strengths. This document analyzes the community dynamics and its reality as phenomenon of endogenous transformation and proposes to strengthen its communication and social participation in order to initiate processes of change and convergence with local and national public policies and actions.

KEYWORDS: COLOMBIA * SOCIAL DEVELOPMENT $*$ COMMUNITIES $*$ COMMUNITY PARTICIPATION * DEVELOPMENT POLICY * COMMUNICATION

Departamento de Comunicación de la Pontificia Universidad Javeriana, Bogotá, Colombia. janneth.palacios@javeriana.edu.co 


\section{INTRODUCCIÓN}

El abordaje de las problemáticas de una comunidad $^{1}$, implica una aproximación a su contexto social, económico, político y cultural, así como, la comprensión de sus dinámicas de cambio y cómo estas determinan formas propias de pensar, de ser $y$ de actuar (Vizer y Carvalho, 2009). Este artículo muestra los principales resultados del diagnóstico realizado a la comunidad del barrio El Dorado en Bogotá, Colombia, en el año 2013, a partir de sus aspectos sociales, económicos, culturales y ambientales como aproximación a su realidad. El diagnóstico a la comunidad, más que una herramienta, es un proceso de participación $y$ reflexión que intenta acercarse a una situación específica para comprender las dinámicas que se desarrollan y las personas que forman parte de este proceso (Davis, 1992), identificar problemas y descubrir potencialidades que les permitan transformar su realidad. Así, la primera sección muestra la metodología $y$ las técnicas aplicadas; posteriormente, se hace la caracterización de la comunidad del barrio desde aspectos sociales, económicos, culturales y ambientales, comparándolos con algunas cifras nacionales. Luego, se plantean las principales problemáticas identificadas por la comunidad, se exponen debilidades $y$ fortalezas y se revisan aspectos de entorno en tanto oportunidades $y$ amenazas.

Con los resultados obtenidos se reflexiona sobre posibles soluciones a las problemáticas sociales de la comunidad, a partir de alternativas de autodesarrollo y se propone el aprovechamiento de sus potencialidades, competencias $y$ aprendizajes para generar acciones

1 Comunidad entendida como un conjunto de personas que comparten elementos en común (sentido de comunidad), por lo general viven en una misma zona geográfica y en su mayoría, se identifican como pertenecientes a un mismo grupo; desarrollan un modo de vida y construyen un conjunto de relaciones recíprocas y solidarias, lo que las hace complejas (Bautista 2012). que permitan mejorar sus condiciones de vida, haciendo énfasis en el papel de las instituciones públicas para asegurar estos procesos, puesto que en las comunidades -en particular, las de barrio-, aun cuando exista la necesidad de auto organizarse para generar dinámicas de autodesarrollo, dependen de los potenciales de desarrollo endógeno del territorio en el que se inscriben, en este caso, la ciudad $y$ de las posibilidades que ofrece el portafolio de políticas públicas, locales y nacionales, que pueden impulsar su dinámica y transformar positivamente su realidad en el corto, mediano y largo plazo, dado que las políticas públicas de los dos niveles (locales y nacionales) inciden en todas las comunidades, así las acciones locales son las que tienen relación más inmediata y directa con la cultura y la sociedad de barrio.

Dentro de la reflexión de resultados se incluye la perspectiva de Amartya Sen -Premio Nobel de Economía en 1998- y su enfoque de desarrollo como expansión de las libertades. Luego, se contrasta la situación de la comunidad del barrio con algunos ejemplos de otras realidades sociales, sin entrar a profundizar en estas comparaciones que pueden ser abordadas en investigaciones posteriores. Finalmente, se proponen algunas líneas de acción dando prioridad a procesos de comunicación y participación.

\section{METODOLOGÍA}

La metodología empleada para esta investigación fue de tipo analítico-descriptiva buscando conocer las características de la realidad de la comunidad del barrio El Dorado, sus problemáticas y necesidades, de dónde emergen y lograr una aproximación a su realidad, comprendiendo sus conexiones $y$ en qué forma sus integrantes desde sus potencialidades, pueden participar en su solución y transformación social (Fernández, 2000). Para desarrollar el trabajo de campo se utilizaron técnicas que involucraron activamente a diferentes grupos y representantes de la comunidad en la identificación de: a) necesidades y problemas que impiden el desarrollo 
de la comunidad, que afectan su cotidianidad y su calidad de vida; b) potencialidades: recursos y fortalezas que posee la comunidad que de manera mancomunada, pueden representar soluciones; c) oportunidades: que son aspectos de orden externo a la comunidad que pueden representar un beneficio y posibilidades de mejora a su calidad de vida $y$ d) amenazas que constituyen situaciones, hechos o circunstancias que pueden afectar o poner en riesgo a la comunidad (Iovanovich y Alurralde, 2007).

Para lograr lo anterior, se plantearon las siguientes etapas:

$\diamond \quad$ Formulación de la metodología de intervención: los datos primarios eran insuficientes, lo que condujo a desarrollar un trabajo de campo amplio para obtener información que permitiera caracterizar la población del barrio. Sin embargo, el escepticismo de la comunidad frente a estos procesos fue el primer obstáculo para arrancar, lo que implicó plantear diferentes actividades para lograr su interés, confianza y participación.

$\diamond \quad$ Exploración: reconocimiento del área y acercamiento a la comunidad: esta etapa buscó conocer el territorio, identificar líderes y organizaciones presentes en el barrio y presentar el trabajo a la comunidad. Para esto, se realizó una actividad cultural como espacio relacionante con la comunidad y se planteó como objetivos: a) generar un espacio lúdico $y$ de integración para los habitantes del barrio; b) sensibilizar a la comunidad sobre el proceso y socializar el trabajo a desarrollar; c) identificar de manera exploratoria, a través de un mural de imágenes y una consulta escrita, cuáles son los aspectos prioritarios por mejorar en el barrio. A esta actividad de imágenes se le llamó "Mural de situaciones, lugares $y$ actores: lo que me gusta de mi barrio $y$ lo que me gustaría cambiar". $\diamond \quad$ Caracterización e identificación de problemáticas: esta etapa tuvo como propósito caracterizar a la población objeto de estudio y lograr el conocimiento más detallado de sus problemáticas y potencialidades a partir de técnicas como la observación participativa: a) entrevistas semi-estructuradas individuales y grupales a líderes de la comunidad y a miembros de organizaciones presentes en el barrio en las que participaron líderes religiosos (Jesuitas y Carmelitas Vedrunas), líderes sociales y juveniles, habitantes con mayor antigüedad en el lugar, representantes de organizaciones sociales, el colegio y miembros de la fuerza pública, entre otros; b) encuesta semi-estructurada para identificar las principales características de los habitantes desde aspectos sociales, económicos, culturales, entre otros, participaron 42 familias, a través de adultos 0 jefes de hogar; c) talleres y focus group: estas actividades tuvieron el propósito de generar una situación de diálogo con grupos específicos (población infantil, adolescente $y$ adultos) para conocer sus expectativas, percepciones y problemáticas sobre el barrio. Se emplearon figuras de apoyo como referente icónico para identificación de temas; actividades lúdicas $y$ talleres con los niños y adultos; se realizó un mural en el que se construían frases que identifican situaciones, lugares y personas de importancia para la comunidad.

En el diagnóstico participaron 159 personas del barrio El Dorado (ver tabla 1), elegidos mediante muestreo no probabilístico, dado que se buscaban personas con un conocimiento amplio sobre el barrio, ya fuera por su experiencia o rol dentro de la comunidad, o por su antigüedad $y$ a partir de esto, identificar nuevos grupos. 
TABLA 1

PARTICIPANTES EN EL DIAGNÓSTICO

BARRIO EL DORADO, COLOMBIA

NOMBRE DEL GRUPO POBLACIONAL

PARTICIPANTES

\begin{tabular}{lc}
\hline Líderes sociales & 6 \\
Mujeres líderes Huerta Casera (amas de casa y jefes mujeres cabeza de hogar) & 7 \\
Líderes religiosos (comunidad Jesuita y Carmelitas Vedrunas) & 7 \\
Habitantes del barrio (adultos, o jefes de familia, hombres y mujeres) & 80 \\
Jóvenes (entre 13 y 18 años) & 5 \\
Niños y niñas (entre 8 y 12 años) & 44 \\
Directivas de la Asociación de Mujeres y Madres & 4 \\
Directiva de la Corporación de Jóvenes & 1 \\
Presidente de AsoJuntas & 1 \\
Miembros del Centro de Atención Inmediata (CAI), El Dorado & 4 \\
\hline
\end{tabular}

Fuente: Elaboración propia.

\section{CARACTERIZACIÓN DE LA COMUNIDAD}

El Dorado es un barrio ubicado en la Localidad $^{2}$ de Santa Fe, en la ciudad de Bogotá, Colombia, en las laderas de los Cerros Orientales. Surgió a finales de los años 70, producto de la venta formal de lotes de una amplia finca, razón por la cual este fue uno de los primeros barrios constituidos legalmente en la ciudad, lo que a su vez facilitó luego el acceso a los servicios públicos (Fundación Pro-educación de El Salvador-FunPRoEs, 2007). La población proviene de diferentes regiones de Colombia por motivo de desplazamiento o por la búsqueda de mejores oportunidades. Al año 2013, el número de habitantes se estimaba en cinco mil, aunque es de anotar que esta cifra puede cambiar dado el traslado constante de los habitantes hacia el centro de la ciudad por razones de trabajo o estudio principalmente. Las edades en promedio

2 Las localidades son definidas por la ley colombiana como divisiones administrativas que poseen una relativa homogeneidad, en cuanto a aspectos geográficos, culturales, sociales y económicos de los distritos especiales (Ley 768 de 2002). En Bogotá existen veinte localidades que a su vez, agrupan varios barrios ubicados en el caso urbano de la ciudad. El Dorado es uno de los 42 barrios de la Localidad de Santa Fe. son de 36 años en adultos, 12 años en niños y 65 años en ancianos (Entrevista, Carlos Buitrago, Barrio El Dorado-Bogotá, 2013).

1. Educación: se identificó que el $50 \%$ de la población cuenta con al menos educación primaria, $25 \%$ con secundaria, $12 \%$ posee educación universitaria y el $13 \%$ no tiene ningún nivel de escolaridad. Estos datos contrastan con la tasa de analfabetismo a nivel nacional que para el año 2012, fue de 5,9\% y en las cabeceras municipales fue del $3,8 \%$ y es comparable con el promedio de analfabetismo registrado en las zonas rurales que para el año 2012 era del 13,3\% (Departamento Administrativo Nacional de Estadísticas-DANE, 2013). Esta situación muestra el nivel de retraso de las comunidades urbanas en materia de educación y la persistencia en las ciudades de los rezagos estructurales heredados de las zonas rurales.

2. Aspectos laborales: se evidenció que el $50 \%$ de la población se encuentra empleada formalmente, un $10 \%$ con subempleos (o empleos menores de 
bajo pago) y un $40 \%$ en situación de desempleo; esta última es una cifra social $y$ políticamente alarmante que afecta las posibilidades de construir comunidad $y$ autodesarrollo. Se observó también que varios de los habitantes que trabajan en ventas informales se desplazan al centro de Bogotá, en particular, a los barrios de mayor concentración de comercio informal (San Victorino y Las Nieves) $y$ otro tanto, desarrollan actividades informales como fabricación de productos de piñatería. La tasa de desocupación $(40 \%)$ es un indicador elevado incluso si se compara con las registradas en el año 2012 en todo Bogotá, cuyo promedio fue de 9\% y en el nivel nacional de 9,6\% (DANE, 2013). Esta situación representa una preocupación importante para la comunidad y es causal de otras problemáticas sociales como el riesgo que niños y jóvenes abandonen sus estudios para dedicarse a trabajar y cubrir necesidades de sus familias, según lo demuestran diferentes investigaciones empíricas (Segovia, 2012), de ahí que los niveles de deserción en educación básica primaria y secundaria se hacen más evidentes en poblaciones de bajos ingresos.

En cuanto a las actividades laborales se encontró que las mujeres son amas de casa o prestan el servicio doméstico y los hombres se dedican al comercio, algunos son pensionados $y$ otros se mueven en oficios varios. Se observó la proliferación de negocios pequeños (microempresas) que son principalmente misceláneas, mini mercados y restaurantes; surgen como mecanismo para la obtención de ingresos adicionales para los hogares $y$ como respuesta al creciente desempleo. Se encontró que el ingreso mensual de la mayoría de las familias (64\%) está entre 0,25 y 2 salarios mínimos mensuales que corresponde a 589.500 pesos en Colombia (2014), lo que equivale aproximadamente a entre
US $\$ 71,9$ y us $\$ 575,4$ mensuales (entre US $\$ 2,3$ y us $\$ 19,1$ diarios) por familia, destacándose que estos ingresos se logran con el salario de dos o más de sus miembros.

3. Tipos de vivienda y formas de edificación: se evidenció que el $84 \%$ son en ladrillo y concreto, siendo la mayoría producto de autoconstrucción. Cuentan con entre 2 y 3 niveles. Su función es principalmente habitacional aunque algunas emplean el primer nivel para el funcionamiento de locales comerciales. En relación con los tipos de viviendas se pudo identificar que el $36 \%$ de los habitantes vive en arriendo, un $47 \%$ en propiedad y un $17 \%$ en vivienda familiar.

4. Aspecto nutricional: se evidenció que la mayoría de la población consume por lo menos 3 raciones de alimentos por día y tan solo un 5\% toma solo una; sin embargo, esta situación es coyuntural. Hasta hace algunos años, el porcentaje de desnutrición en el barrio era alto, niños, jóvenes e incluso ancianos, presentaban problemas de mal nutrición de acuerdo a María Dolores Acequinolaza, religiosa de la Comunidad Carmelitas Vedrunas, quien desde hace más de 30 años desarrolla proyectos sociales para los barrios el Dorado y el Consuelo (entrevista, 2013). En los últimos años la situación ha cambiado, principalmente, por las políticas de asistencialismo social del gobierno a través de comedores infantiles $y$ la entrega de mercados. Aunque en este momento, el aspecto nutricional no es un punto crítico de la situación de la comunidad, existe preocupación respecto a qué pasará cuando se acaben las políticas sociales de asistencialismo a poblaciones vulnerables. También es de anotar que existe inconformidad por parte de la comunidad respecto a la cobertura de los comedores comunitarios, dada la alta demanda que 
estos tienen y los criterios para acceder a este beneficio (Secretaria de Salud, Alcaldía Mayor de Bogotá, 2013).

5. Cobertura de servicios: en lo que corresponde a cobertura de servicios de salud, el $89 \%$ de la población está integrada a algún tipo de sistema de seguridad social; aunque la comunidad manifiesta que el servicio es deficiente $y$ no acuden a este por la demora en la atención o por la distancia, salvo casos puntuales o de máxima gravedad. Por otra parte, se destacó que la población cuenta con cubrimiento de servicios públicos: $100 \%$ energía y agua, $77 \%$ gas natural y $95 \%$ recolección de basuras. Para los habitantes es motivo de orgullo mostrar que la llegada de estos servicios fue producto del interés colectivo y la presión a los gobiernos locales.

Si bien, estos últimos datos pueden mostrar logros importantes de satisfacción de necesidades básicas, en el futuro inmediato las presiones de las comunidades de menores ingresos estarán orientadas hacia otros temas que les permitan disponer de condiciones superiores para competir en el mercado laboral, desarrollar su potencial de emprendimiento $y$ escalar de nivel socio económico. En otras palabras, la presión social, como se está observando en otras economías emergentes, por ejemplo, en Brasil y Chile, apuntan a reivindicaciones de calidad $y$ de oportunidades superiores de desarrollo, que implican una ostensible mejora en los sistemas educativos, de participación, de inclusión laboral, entre otros.

6. Aspectos de seguridad: de acuerdo con información obtenida a través de la entrevista con Carlos Buitrago (el Dorado, Bogotá, 2013), al mes de abril del año 2013, los delitos que con mayor frecuencia se presentan en el barrio son las lesiones comunes y el hurto a personas. Se indicó que en este mes los casos reportados son bajos comparados con los índices de otros barrios de la misma localidad en Bogotá, igualmente se informó que los delincuentes no operan en el mismo sector sino que se desplazan a otras zonas. En lo que se refiere a las acciones de seguridad de la policía en el Barrio, se identificó que existe poca credibilidad de la comunidad respecto a su eficacia. La población indicó que poco les interesa $y$ poco participan en las campañas que promueven los agentes de policía. Es así como el $47 \%$ de la población encuestada indicó que no tienen confianza y un $21 \%$ tienen poca confianza en las acciones de la fuerza pública. Algunos de los motivos mencionados fueron la demora en respuestas y la falta de control sobre el micro tráfico de sustancias psicoactivas.

7. Aspectos psicosociales: desde la perspectiva de los habitantes consultados en la encuesta, el $74 \%$ perciben que el consumo de sustancias psicoactivas (SPA) es el principal problema e incide en la generación de delitos e inseguridad entre los habitantes. También se expresó preocupación por el incremento en el consumo de estas sustancias por parte de menores y se pidió protección especial para ellos.

En la gestión realizada por los agentes de policía se encontró que el control en el consumo de alucinógenos -medido por la cantidad de gramos decomisados-, al mes de abril del año 2013, había aumentado en un $66 \%$ comparado con el total del año 2012, mostrando una clara relación con la percepción que tiene la comunidad respecto al incremento tanto en el consumo como en el comercio de sustancias psicoactivas.

El consumo de estas sustancias es una situación que obedece a diferentes razones y se presenta en todas las edades. Algunos de los factores que más inciden en que los jóvenes hagan uso de estas tienen que ver con el bajo rendimiento 
académico, la actitud favorable frente al consumo, la ausencia de metas, tener autoimagen negativa, poca tolerancia al estrés, poca asertividad, la influencia de pares, personalidad dependiente, impulsividad y el fácil acceso a las sustancias debido a las características del sector (Secretaría Distrital de Integración Social, 2010).

Los datos presentados muestran una situación desalentadora para la comunidad; sin embargo, es de anotar que experiencias recientes en otras comunidades quizá en situaciones más complejas como en las favelas en Rio de Janeiro en Brasil, donde la violencia de todo tipo azotó a sus habitantes, luego de un largo periodo intentando construir comunidades de barrio, lograron su propia autorregulación, acordando reglas de juego con el apoyo de instituciones locales, para que la violencia desapareciera de su territorio, al punto que estos lugares hoy no solo cuentan con una mejor convivencia entre sus habitantes sino que incluso, se están promoviendo actividades de turismo que ofrecen la posibilidad al visitante de alojamiento por temporadas largas.

8. Aspectos asociativos y de comunicación: del aspecto asociativo se encontró que la población de El Dorado se organiza para acciones o problemáticas puntuales $y$ pocas personas sostienen una vinculación permanente con alguna organización. Los principales proyectos en los que la comunidad ha intervenido son la construcción de la Parroquia San Alberto Hurtado (SAH), la creación del teatro al aire libre y la huerta casera. A su vez, los grupos en los que algunos de los habitantes participan son: Pastoral de la Parroquia, actividades promovidas por la Asociación de Mujeres y Madres $y$ en menor nivel, las que organiza la Junta de Acción Comunal.

Por otra parte - aunque en el Barrio existen diferentes mecanismos de comunicación y participación-, hay ausencia de escenarios de encuentro donde la comunidad pueda presentar sus ideas, exponer sus problemáticas y consolidar dinámicas de integración. No obstante, se logró observar que la comunicación entre los habitantes está marcada por la solidaridad y el respeto. Las personas del Barrio son amables con los visitantes y solidarias con los que llegan a asentarse. Consideran que su espíritu solidario les ha permitido convivir, aun cuando la población en los últimos años ha cambiado y crecido producto de la problemática social del país. Existe un adecuado ambiente para escuchar iniciativas externas, aunque la comunidad espera resultados rápidos y efectivos.

De la comunicación entre las organizaciones presentes en la comunidad, se muestra como efectiva y cercana. Estas organizaciones, en su mayoría de orden no gubernamental, han sabido instaurarse en el territorio apoyando la solución a problemáticas sociales $y$ manteniendo un vínculo con la comunidad a través de los diferentes proyectos que desarrollan; los líderes de estas organizaciones son habitantes del Barrio y esto les ha permitido no solo conocer con mayor profundidad las condiciones de su comunidad, sino vivirlas y ser parte de estas.

En contraste, la comunicación de la comunidad con las entidades públicas, administraciones locales $y$ otras, no es percibida como eficiente. Existe una sensación de escepticismo frente a lo que estas hacen; esto como consecuencia de la forma como algunos políticos se han interesado en la comunidad, quienes han llegado en busca de beneficios electorales sin verdaderas soluciones a sus necesidades, según manifestó la población del Dorado.

9. Situación ambiental : para finalizar esta caracterización, en lo que corresponde a las condiciones ambientales se observó que el Barrio debido a estar ubicado en las laderas de los Cerros Orientales, goza 
de una amplia y atractiva panorámica $y$ está rodeado por amplias zonas verdes. Los habitantes indican que el sector es privilegiado dado que se aleja de la contaminación del centro de la ciudad y el entorno hace que los niños crezcan en un ambiente rodeado por la naturaleza. Sin embargo, varios de los vecinos han manifestado que algunas de sus zonas se han deteriorado por la presencia de consumidores de sustancias psicoactivas, el mal uso de los recursos, la falta de recolección de la basura, el poco control de las mascotas que escudriñan la basura y ensucian los lugares comunes y la ausencia de una mayor conciencia social.

A lo anterior se le suma que según el informe del Hospital Centro Oriente (HCO), El Dorado se encuentra en una zona de riesgo ambiental que presenta como posibles situaciones: fenómenos de derrumbe, inundación, riesgo sísmico, colapso estructural, incendios estructurales $y$ forestales $y$ desbordamientos (HCO, 2011) y aunque la población es consciente de estos peligros, varias familias se instalan en el lugar por necesidad, haciendo caso omiso a estas condiciones.

\section{PRINCIPALES PROBLEMÁTICAS Y NECESIDADES DE LA COMUNIDAD}

Producto de las diferentes actividades de interacción realizadas con la comunidad $y$ de los datos anteriormente esbozados, se identificaron problemáticas presentes y necesidades manifiestas por los habitantes del barrio El Dorado como las que se presenta a continuación. Se agrupan dentro de aspectos socioeconómicos, culturales, laborales y psicosociales y se plantea un análisis que identifica debilidades, fortalezas o potencialidades detectadas y posibles oportunidades con que cuenta la comunidad y que pueden aportar en la solución de las problemáticas y finalmente, se exponen algunas amenazas que pueden aumentar la incidencia de los problemas identificados.
1. ASPECTOS SOCIO ECONÓMICOS Y CULTURALES: ANALFABETISMO, DESERCIÓN ESCOLAR, BAJO NIVEL ACADÉMICO EN ESTUDIANTES DE PRIMARIA Y SECUNDARIA, AUSENCIA DE OPORTUNIDADES PARA ACCEDER A EDUCACIÓN SUPERIOR.

\section{Debilidades}

$\diamond \quad$ Existe en el barrio un solo Colegio (público) para atender el creciente número de estudiantes en los grados de primaria y secundaria, así como pocos jardines para la formación a la primera infancia.

$\diamond \quad$ Existe una biblioteca en funcionamiento (barrio El Consuelo) que presta el servicio; no obstante, presenta limitación de recursos (personal, espacio, tecnología y mobiliario).

$\diamond \quad$ La mayoría de la población adulta cursó únicamente primaria y cerca del $13 \%$ es analfabeta.

$\diamond \quad$ Los estudiantes de primaria y secundaria presentan dificultades académicas en áreas como matemáticas, español e inglés.

$\diamond \quad$ Los niños, jóvenes $y$ adultos requieren fortalecer su capacitación en ofimática y en tecnologías de información y comunicación. La mayoría de las familias no cuentan con acceso a Internet.

$\diamond \quad$ La situación económica conlleva a que los jóvenes dejen sus estudios para buscar ingresos para sus familias, lo cual aumenta la posibilidad de deserción escolar.

$\diamond \quad$ Faltan programas $y$ estrategias de formación en educación sexual y reproductiva.

$\diamond \quad$ Ausencia de programas de formación para el trabajo directamente con el Barrio.

$\diamond \quad$ Las organizaciones sociales presentes en el lugar no cuentan con suficientes recursos para desarrollar sus actividades y apoyar a la comunidad.

$\diamond \quad$ La comunidad posee poca información sobre programas de educación formal e informal ofrecidos por entidades públicas. Por otro lado, desconocen las oportunidades que ofrecen diferentes entidades para impulsar proyectos sociales, productivos, culturales y de educación, entre otros. 


\section{Oportunidades}

$\diamond \quad$ En el país se ofrecen programas de educación técnica y tecnológica de acceso gratuito impartidos por entidades públicas como el Servicio Nacional de Aprendizaje (SENA).

$\diamond \quad$ Existe interés por parte de entidades de educación superior de aportar en procesos de educación a la comunidad y por parte del sector productivo, para incrementar la mano de obra calificada en el país y desarrollar diferentes sectores de la sociedad.

$\diamond \quad$ Las tecnologías de comunicación e información permiten diversificar el acceso a la educación.

$\diamond \quad$ Existen programas de financiamiento por parte del Estado $y$ entidades privadas para acceder a educación superior.

\section{Fortalezas}

$\diamond \quad$ Se hallan en el Barrio organizaciones sociales que apoyan la solución de problemáticas de la comunidad.

$\diamond \quad$ La mayoría de la población infantil y adolescente tiene interés en mejorar su nivel académico.

$\diamond \quad$ Las personas están interesadas en ayudar, pero no cuentan con la suficiente formación.

$\diamond \quad$ La ubicación del Barrio, el recibir capacitación en otros lugares y contar con un servicio de transporte público permanente.

\section{Amenazas}

$\diamond \quad$ Aumento de la deserción escolar y en el bajo nivel académico de los estudiantes de primaria y secundaria.

$\diamond \quad$ Ausencia de oportunidades para acceder a educación superior.

$\diamond \quad$ El bajo nivel educativo reduce posibilidades de inserción laboral, incrementa el desempleo $y$ aumenta la informalidad.

$\diamond \quad$ Situaciones del entorno pueden afectar el interés y la continuidad del proceso educativo, como el consumo de drogas, bajos ingresos y embarazos a temprana edad.

$\diamond \quad$ Aumento en la desigualdad social.
2. ASPECTOS SOCIOECONÓMICO Y LABORAL: ALTO NIVEL DE DESOCUPACIÓN, PROLIFERACIÓN DE EMPLEOS INFORMALES Y BAJOS INGRESOS POR FAMILIA.

\section{Debilidades}

$\diamond \quad$ Ni en el Barrio ni el sector se ofrecen oportunidades laborales que brinden estabilidad a la comunidad.

$\diamond \quad$ La situación de desplazamiento y en general, la situación social del país incrementa el nivel de desocupación y el bajo nivel de ingresos por familia.

$\diamond \quad$ El bajo nivel de escolaridad en la población hace que la mano de obra calificada sea escasa.

$\diamond \quad$ Existe una alta informalidad laboral en el barrio.

$\diamond \quad$ Escasa presencia de entidades comerciales, financieras y de promoción al comercio formal.

$\diamond \quad$ Existe desconocimiento por parte de la comunidad de programas de apoyo al emprendimiento $y$ al desarrollo empresarial.

$\diamond \quad$ Falta de mayor conciencia por parte de la comunidad respecto al ahorro.

$\diamond \quad$ Falta de organización comunitaria para crear proyectos productivos $y$ falta de visión de negocios de largo plazo.

\section{Oportunidades}

$\diamond \quad$ Generación de alianzas interinstitucionales para apoyar iniciativas o capacitar en temas empresariales y de formación laboral.

$\diamond \quad$ El sector productivo y la sociedad requieren personas para diferentes actividades.

$\diamond \quad$ Existen organismos internacionales orientados a apoyar el desarrollo económico y social en población de escasos recursos.

$\diamond \quad$ Existe interés por parte de instituciones educativas para fortalecer $y$ orientar planes de emprendimiento y desarrollo socio económico.

$\diamond \quad$ El gobierno ofrece acompañamiento $y$ orientación técnica para iniciativas productivas y posibilidades de financiación para micro $y$ pymes, lo cual facilita la creación de negocios. 


\section{Fortalezas}

$\diamond \quad$ Cerca del $45 \%$ de la población del barrio se encuentra en edades económicamente activas, tanto hombres como mujeres.

$\diamond$ Existe un amplio número de microempresas, algunas formalmente constituidas $y$ que funcionan en el barrio como respuesta a la necesidad de mejorar los ingresos a corto plazo.

$\diamond \quad$ Existen proyectos iniciados por las Organizaciones No Gubernamentales y las organizaciones sociales que funcionan en el Barrio, los cuales podrían ser fortalecidos para ampliar su impacto.

$\diamond \quad$ Facilidad en el acceso al Barrio y cercanía con el centro de la ciudad, así como, servicio de transporte permanente que facilita el desplazamiento a otros sectores de la ciudad.

$\diamond \quad$ La comunidad es entusiasta, perseverante y con espíritu emprendedor.

\section{Amenazas}

$\diamond \quad$ Delincuencia e inseguridad como resultado de la falta de empleo y de oportunidades.

$\diamond \quad$ Incremento de la informalidad en actividades económicas.

$\diamond \quad$ Aumento en el micro tráfico de sustancias psicoactivas, dada la rapidez con que se obtienen ganancias y al incremento en el consumo.

$\diamond \quad$ Cercanía con sectores de alto nivel de comercialización y consumo de sustancias psicoactivas.

3. ASPECTO SOCIOECONÓMICO Y PSICOSOCIAL: MICRO TRÁFICO DE SUSTANCIAS PSICOACTIVAS Y AUMENTO EN EL CONSUMO.

\section{Debilidades}

$\diamond \quad$ Alto tráfico de sustancias psicoactivas (SPA) en lugares cercanos al colegio $y$ en diferentes espacios públicos, así como presencia de consumidores en estos lugares.

$\diamond \quad$ El consumo de sustancias psicoactivas se presenta en todas las edades, aunque se hace más evidente en población adolescente. $\diamond \quad$ Grupos de jóvenes en pandillas para comercialización y consumo de SPA.

$\diamond \quad$ Influencia de grupos sociales en hábitos de consumo en población infantil y jóvenes.

$\diamond \quad$ Poco interés hacia el deporte, la cultura y la recreación.

$\diamond \quad$ Falta de asesoría para la prevención del consumo de drogas y una mayor orientación hacia la construcción de un proyecto de vida.

$\diamond \quad$ Falta de ayuda profesional a personas consumidoras de SPA.

$\diamond \quad$ Poco interés del grupo familiar frente a personas consumidoras en el mismo grupo.

$\diamond \quad$ La ubicación del Barrio facilita la entrada de este tipo de sustancias, puesto que existe cercanía con sitios de alta comercialización y consumo.

$\diamond \quad$ Temor y poca acción de la comunidad para enfrentar esta problemática.

\section{Oportunidades}

$\diamond \quad$ Existencia de centros de ayuda profesional para la rehabilitación de personas que consumen SPA en diferentes lugares de la ciudad.

$\diamond \quad$ Generación de acciones desde la comunidad para evitar esta situación.

$\diamond \quad$ Mayor preocupación del grupo familiar por el bienestar de sus miembros $y$ ante personas que de su propio núcleo familiar presenten esta situación.

$\diamond \quad$ Mayores estrategias de prevención, tanto en los colegios como en las diferentes organizaciones presentes en la comunidad.

$\diamond \quad$ Generación de mayores oportunidades laborales y educativas para jóvenes $y$ adultos.

$\diamond \quad$ Fortalecimiento de actividades deportivas, de recreación y culturales que promuevan el uso de tiempo libre y hábitos de vida saludable.

$\diamond \quad$ Mayor presencia y acción de la autoridad y participación ciudadana apoyando la seguridad en el Barrio, así como, mayores campañas de salud y prevención.

$\diamond \quad$ Mayores actitudes $y$ comportamientos responsables de los padres $y$ adultos 
frente a su grupo familiar. Mayor comunicación entre la familia, su comunidad $y$ las organizaciones que pueden aportar a la solución de este problema.

$\diamond \quad$ Mejorar el conocimiento de los habitantes $y$ del entorno que rodea a la familia, a los jóvenes y a los niños.

\section{Amenazas}

$\diamond \quad$ Delincuencia e inseguridad como resultado del consumo de SPA.

$\diamond \quad$ Creciente aumento en el micro tráfico de SPA e incremento en el número de consumidores de estas sustancias, especialmente, en adolescentes.

$\diamond \quad$ Desinterés en desarrollar un proyecto de vida.

\section{ANÁLISIS DE LOS RESULTADOS}

De todo lo anterior, se logra observar que las diferentes situaciones identificadas en la comunidad están relacionadas entre sí y ameritan intervención oportuna, efectiva y conjunta por parte de las entidades de gobierno $y$ otras organizaciones, como de la misma comunidad. Aspectos como la falta de mayores oportunidades para jóvenes, incluso también para adultos, conlleva a otras problemáticas como son la inseguridad, el consumo de sustancias psicoactivas, la informalidad y el desempleo, entre otras. Sumado a lo anterior, la posibilidad de aumentar la deserción escolar, como resultado de la necesidad de los estudiantes de buscar oportunidades laborales para aportar ingresos a sus familias, algunos dedicándose a actividades informales o al comercio de drogas como mecanismo para obtener ganancias de manera rápida. Junto con la deserción, la baja calidad educativa que se evidenció con el bajo rendimiento académico de los estudiantes de primaria y secundaria, constituye otra preocupación expresada por la comunidad, lo cual representa la reducción de posibilidades para acceder a educación superior y para inserción al mercado laboral, aumentando la situación de pobreza y de desigualdad.

Al respecto, Amartya Sen, Nobel de Economía en 1998,indicó que la pobreza es la privación de las capacidades básicas en la población tales como educación, salud, seguridad y no solo la falta de ingresos (renta) como se ha considerado tradicionalmente (2000: 114); sin embargo, afirmó que estos dos aspectos están directamente relacionados, puesto que un aumento en las capacidades, no solo aumenta la calidad de vida, sino que posibilita que una persona pueda ganar o mejorar sus ingresos (2000: 118). En consecuencia, la situación de pobreza como resultado no solo de los bajos ingresos, sino de la falta de oportunidades laborales, la baja calidad de la educación y las condiciones de inequidad social a la que los habitantes de este barrio se enfrentan en su cotidianidad y que se evidenció por el nivel de desempleo (40\%), resulta ser otra situación crítica que requiere solución inmediata. Asimismo, Sen menciona que:

... en los países en vías de desarrollo en general, es fundamental la necesidad de adoptar iniciativas públicas para la creación de oportunidades sociales... los países que hoy son ricos poseen una historia bastante notable de medidas públicas relacionadas con la educación, la asistencia sanitaria, la reforma agraria, etc. La amplia difusión de estas oportunidades sociales permitió a la mayoría de los individuos participar directamente en el proceso de expansión económica (2000: 180).

La perspectiva de Sen remite no solo a la necesidad de satisfacer condiciones básicas sino también a la libertad de las personas de expresarse $y$ de participar libre, amplia $y$ activamente en lo económico, cultural y político; no obstante, las bases para avanzar en este sentido está en las políticas de desarrollo de largo plazo de la nación y del territorio, sobre todo en educación, ciencia y tecnología y desarrollo productivo, como complemento a las inversiones en infraestructura de distinto tipo, conduciendo a la formación de instituciones más inclusivas y con mayor compromiso con el desarrollo de su población (Acemoglú y Robinson, 2012).

Como se observó en los resultados, la preocupación por mejorar los ingresos hace que familias del barrio se concentren en atender las necesidades diarias, algunas crean negocios 
informales, con visión de corto plazo y precaria perspectiva administrativa; otras se desplazan a diferentes zonas de la ciudad a emplearse en servicios domésticos, de construcción o en ventas informales. Como resultado, los núcleos familiares pierden unión y cercanía, situación que induce a que la población infantil y los jóvenes sean más vulnerables a las problemáticas del Barrio. Asimismo, las acciones en materia de desarrollo son insuficientes en la comunidad, por ejemplo, no existen programas que promuevan el emprendimiento o la organización comunitaria para construir proyectos productivos; la situación de desempleo en el Barrio y en el país se hace prolongada, lo cual a su vez aumenta la exclusión social, en tanto que las personas pierden los beneficios $y$ las prestaciones sociales que otorga una vinculación laboral formal.

Sen afirma que la denegación o falta de la libertad para participar en el mercado laboral es también una forma de esclavitud, presente hoy en muchos países y sostiene que para "aprovechar las oportunidades que ofrece el comercio mundial que se basan en una cada vez en mayor exigencia en calidad, innovación y eficiencia, resulta difícil que lo comprendan, logren y mantengan trabajadores que no saben leer y escribir ni calcular" (2000: 181). Entonces, se hace evidente que ya no es suficiente lograr amplias coberturas, ni subsidiar de manera abundante y durante largo tiempo a la gente en situación de pobreza. Los programas de ampliación de coberturas y de subsidios, deben ser temporales y cambiantes, así como también estar respaldados en políticas de Estado que eleven el nivel cultural, educativo, económico y político de las personas. Cobertura $y$ calidad son parte de un mismo propósito, pero no se dan ni convergen de manera espontánea. La cobertura para que sea digna implica que sea de calidad, tanto en la prestación de sus servicios $y$ en sus equipamientos, pero se debe considerar que la calidad también depende de otros factores como políticas de Estado en materia industrial y de innovación, de ciencia y tecnología, de desarrollo de infraestructuras y de un nuevo urbanismo, de generación de capacidades de crear y transformar el arte $y$ la cultura, $y$ de liberación del potencial de innovación en la producción, el emprendimiento, la preservación del medio ambiente, el uso y desarrollo de las nuevas tecnologías de la información y las redes de múltiple propósito que posibilite fortalecer el rol de los medios para contribuir a divulgar y transformar la cultura y la cosmovisión del ciudadano, todo con la finalidad de construir instituciones inclusivas: innovadoras, incluyentes $y$ de democracia participativa.

Finalmente, es necesario resaltar que quizás la mayor problemática y el temor más evidente de la comunidad del Barrio es el aumento en el consumo de sustancias psicoactivas. Los habitantes expresan que esta situación empeora con el paso de los días, consideran que los adolescentes son las principales víctimas y solicitan con urgencia mayores medidas de control. Como se observó, el Barrio por su ubicación geográfica está expuesto a este comercio, incluso, se encuentra cerca de lo que popularmente se conoce en Colombia como "ollas" o lugares de expendio, consumo y tráfico de sustancias psicoactivas, condiciones que agudizan el problema.

Aunque es alarmante este comportamiento social en torno al uso y comercio de sustancias psicoactivas, en gran parte es el reflejo de la carencia de oportunidades. Al final ni el Estado ni la sociedad, en sus múltiples intervenciones, llegan para quedarse y cuando se quedan, su aporte o solución no es sostenido.

\section{COMPARACIÓN CON OTRAS REALIDADES SOCIALES}

En países avanzados y en casos similares de territorios habitados por personas con escasos ingresos (considerando que las poblaciones en situaciones precarias son menores $y$ las políticas superiores), las capacidades locales de organización, regulación y autodesarrollo son más relevantes, excepto cuando surgen crisis económicas estructurales que afectan a todas las regiones y a toda la nación, como ocurrió de manera reciente en España y como también ha ocurrido en países de América Latina que han sufrido agudas inflexiones estructurales 
de su economía, como las vividas en Argentina, Brasil y Ecuador, que terminan afectando aún más a la gente pobre.

Es así como el impacto de las políticas públicas se hace evidente, tanto en países en desarrollo y centralizados como Colombia, donde la población en situación de pobreza es grande y se encuentra dispersa en todas las ciudades $y$ regiones. Las políticas nacionales de desarrollo resultan determinantes para impulsar y complementar la acción propia de las comunidades de barrio y las mismas políticas y programas de la ciudad, como también en sistemas político administrativos federales como Brasil, donde su programa "Bolsa Familia" se complementa con otras acciones para sacar de la pobreza a grandes segmentos de población y convertirlos en clase media.

Este es el panorama que está vislumbrándose en América Latina, cuyos resultados son de mediano y largo plazo, porque así como los problemas estructurales se incuban $y$ se consolidan durante décadas y décadas, su solución conlleva agendas sostenidas de largo término, porque construir viviendas, construir vías, adecuar un parque, abrir colegios, un centro de salud o un centro cultural, son equipamientos necesarios pero no son soluciones de fondo y suficientes para lograr desarrollo, por eso se requieren de estrategias, programas y proyectos que sean sostenidos $y$ que propendan por aumentar las capacidades de la población. Al respecto, Amartya Sen (2000) afirma que el desarrollo debe ser concebido como un proceso de expansión de las libertades reales de los seres humanos, en el cual el papel de las instituciones públicas es vital no solo para evitar privaciones como la inanición, desnutrición, entre otros, sino para generar mayores y mejores oportunidades sociales, políticas y económicas.

La situación esbozada se constata en la mayoría de países latinoamericanos, entre ellos Colombia, razón por la cual, los diagnósticos y los ejercicios sobre identificación de problemas, necesidades $y$ oportunidades en poblaciones como la comunidad del barrio El Dorado, no varían de manera importante entre los resultados de una investigación reciente con los de pesquisas realizadas diez, quince o veinte años atrás (ver por ejemplo, el estudio sobre las condiciones de vida de los jóvenes nicoyanos en Costa Rica en 1994; Moreno, 1994).

El problema de falta de oportunidades sostenidas hace que el tejido social esté sometido a la presión vital del diario vivir que lo desliga de procesos sociales sostenidos de desarrollo y de integración social. La evidencia empírica lo muestra, por ejemplo, a pesar de todas las intervenciones del Estado $y$ de los esfuerzos de las mismas comunidades, en Medellín (Colombia) persiste la violencia en sectores pobres como los de las comunas. Por su parte, en Chile, el trabajo adelantado para abatir la pobreza apenas logró resultados luego de veinte años (Programa de las Naciones Unidas para el Desarrollo-PNUD, 2011) y pueden faltar otros diez o quince más para lograr reducir la pobreza a la tasa de un dígito y de ahí avanzar a reducir la inequidad. En Brasil, los dos gobiernos de Lula Da Silva y en el actual de Dilma Rousseff, se han logrado resultados importantes, pero aun deberán transitar por otros años más para lograr reducir la pobreza y la desigualdad social a niveles aceptables y para alcanzar umbrales de desarrollo.

En consecuencia, los logros para disminuir la pobreza de manera estructural que redunden en comunidades tranquilas, solidarias, emprendedoras, innovadoras, más educadas y más productivas son resultado de acciones sistémicas intersectoriales y duraderas. Los subsidios de distinto tipo para acceder a salud, servicios básicos y educación, no son suficientes, son una fase inicial de las políticas sociales. Se requiere de un esfuerzo de todos para aumentar el desarrollo productivo, la educación, la ciencia y la tecnología, las infraestructuras, el medio ambiente, el urbanismo, la seguridad e incluso, el rol de los medios de comunicación, entre otros factores, que son los que hacen posible el ascenso sostenido de las comunidades en el largo plazo. En consecuencia, en Colombia con una industrialización tardía e incompleta y que se encuentra en una etapa de transición, resulta fundamental dar un salto de calidad en sus políticas de desarrollo y en la construcción de instituciones incluyentes para 
nivelar las condiciones de bienestar en una sociedad global que depende cada vez más del conocimiento.

\section{CONCLUSIONES}

La situación que se logró evidenciar en la población del barrio el Dorado, corrobora la precariedad a la que se ven expuestas la mayoría de las familias colombianas y de países de América Latina. Las acciones que ahora impulsan varios de estos países para abatir la pobreza $y$ atenuar la inequidad, solo son acciones de la transición que viven mientras logran una fase superior de desarrollo, la cual puede demorarse varias décadas como se mencionó, porque el ataque a la pobreza y situar a la gente en el status de clase media, debe basarse en el desarrollo de un sistema productivo con educación, ciencia y tecnología, así como con condiciones dignas en seguridad social, pero esos son procesos que demoran muchos años y que requieren de esfuerzos sostenidos que países como Colombia no son afortunados en emprender. Corea o Singapur, llevan más de 60 años de desarrollos continuos y aun no alcanzan los indicadores de los países más avanzados y lo paradójico es que les pueden faltar $20 \mathrm{u}$ otros años más para lograrlo.

Sen sostiene que el desarrollo humano se basa en la creación de oportunidades sociales y que estas inciden en la expansión de las capacidades humanas $y$ en la mejora de la calidad de vida, puesto que:

La expansión de la asistencia sanitaria, la educación, la seguridad social, etc., contribuyen a la calidad de vida $y$ a mejorarla. Existen pruebas de que, incluso con una renta considerada baja, un país que garantiza la asistencia sanitaria y la educación a todos puede conseguir, de hecho, notables resultados en lo que se refiere a longevidad $y$ calidad de vida de toda la población (2000: 181).

Esto parece contrastar hoy con la realidad, más aún cuando la tendencia de las últimas cuatro décadas en el mundo, ha sido la de reducir las condiciones de los modelos sociales de bienestar para impulsar el desarrollo desde la iniciativa individual (el individuo como auto organización) y desde el enfoque de eficiencia $y$ productividad que finalmente ha conllevado a una mayor precariedad de la situación social, laboral y aumenta la brecha de desigualdad.

Por ello, promover cambios a través de acciones conjuntas donde se vinculen diferentes actores de la sociedad que propendan por articular los intereses y necesidades de la comunidad con el desarrollo de la ciudad, de la sociedad y del país, resulta ser para Colombia todo un desafío. Los resultados expuestos son reflejo del deterioro social que sufren gran parte de los colombianos; evidencia la problemática social nacional, muestra que el conflicto no solo está en las zonas rurales $y$ hace latente la falta de atención de las instituciones sociales y económicas que son las llamadas a garantizar $y$ satisfacer las condiciones básicas y de progreso de la sociedad (salud, educación, ciencia, tecnología, arte, cultura, emprendimiento, seguridad, libertades políticas, entre otras). En consecuencia, lograr la transformación de las condiciones aquí enunciadas implica para la comunidad aumentar sus procesos de auto organización, auto gestión y auto producción, lo que significa lograr mayor participación en la construcción de soluciones y compromiso frente a las mismas, así como, la articulación con otras dinámicas de la ciudad, construir redes de colaboración, de comunicación y de aprendizaje con comunidades en similar situación.

La identificación y el reconocimiento de problemáticas $y$ potencialidades es un avance para la comunidad, puesto que no solo aumenta su consciencia sobre estos temas, sino que incrementa el compromiso frente a posibles soluciones, por ello, mejorar la comunicación constituye otro importante reto para articular procesos de participación, así como, la posibilidad de fortalecer su identidad y sentido de pertenencia como grupo social, reafirmando sus valores, creencias y saberes (Gumucio, 2004). Sen propone que las personas deben participar más en las decisiones sociales y en la elaboración de las decisiones públicas a través de los mecanismos institucionales, lo cual no solo es un derecho sino una responsabilidad si en 
realidad se busca una transformación social (2000: 21). Para este autor, el debate público y la participación social son determinantes para la elaboración de la política económica y social en un sistema democrático; sin embargo, se insiste en la convergencia y suma de esfuerzos sostenidos y articulados entre acciones de las comunidades de barrio y las políticas, estrategias, programas y proyectos de la ciudad y del país. Las comunidades solas logran ciertos objetivos, las comunidades con respaldo sostenido tienen propósitos superiores $y$ se plantean mayores objetivos de progreso, con acciones sostenidas y sistémicas de múltiple tipo, debido a lo que las comunidades de barrio, las ciudades de las cuales hacen parte y la nación, son un todo.

\section{BIBLIOGRAFÍA}

LIBROS

Acemoglú, Daron y Robinson, James. Por qué fracasan los países.Barcelona, España: Deusto, 2012.

Fernández, Carlos. La comunicación humana en el mundo contemporáneo. México: McGraw-Hill, 2000.

Sen, Amartya. Desarrollo y libertad. Barcelona, España: Editorial Planeta SA, 2000.

\section{PUBLICACIONES PERIÓDICAS}

Bautista, Rafael. "¿Qué quiere decir comunidad?". Revista de Estudios Bolivianos 19. Pensilvania, Estados Unidos. University Library SystemUniversity of Pittsburgh, 2012: 159-189.

Gumucio Dagron, Alfonso. "El cuarto mosquetero: la comunicación para el cambio social". Investigación y Desarrollo 12 (1). Barranquilla, Colombia. Universidad del Norte, 2004: 02-23.

Iovanovich, Marta y Alurralde, Emilia.“¿Cómo podemos conocer a la comunidad?". Revista Iberoamericana de Educación.[Revista digital]. Centro de Altos Estudios Universitarios de la Organización de Estados Iberoamericanos (CAEU/OEI), 2007: 1-7.
Moreno, Wagner. "Condiciones de vida y su incidencia en la identidad personalsocial de adolecentes nicoyanos". Revista deCiencias Sociales 66. San José, Costa Rica. Editorial de la Universidad de Costa Rica, 1994: 37-44.

Vizer, Eduardo y Carvalho, Helenice. "Comunicación y socio análisis en comunidades y organizaciones sociales". Revista Latinoamericana de Ciencias de la Comunicación 6 (11). Brasil. Asociación Latinoamericana de Ciencias de la Comunicación (ALAIC), juliodiciembre 2009: 149-157.

\section{TEXTOS ELECTRÓNICOS}

Departamento Administrativo Nacional de Estadísticas-DANe. Comunicado de prensa- Encuesta Calidad de Vida 2012. En: <http://www.dane.gov.co/index.php/ es/estadisticas-sociales/calidad-de-vidaecv/87-sociales/calidad-de-vida/4623encuesta-de-calidad-de-vida-2012> [consultado el 13 de marzo de 2013].

Fundación Pro-educación de El Salvadorfunproes. El Dorado Centro Oriental, Reseña barrial. Julio 2007. En: <http:// funprofes.org/santafelocal/resenas/ word/9611.pdf> [consultado el 04 de abril de 2013].

\section{ENTREVISTAS}

María Dolores Acequinolaza. Fundadora de la Comunidad Hermanas Carmelitas Vedrunas. Entrevista realizada en el Barrio El Consuelo, Bogotá, Colombia. 20 de abril de 2013.

Carlos Buitrago. Auxiliar de información. Centro de Atención Inmediata-CAI. Entrevista realizada en el Dorado, Bogotá, Colombia. 22 de abril de 2013.

OTROS

Davis Case, D’Arcy. "Conceptos, métodos $y$ herramientas para el diagnóstico, seguimiento y la evaluación participativos en el desarrollo forestal 
comunitario". Manual de Campo. Roma: Organización de las Naciones Unidas, Food and Agriculture Organization-ONUFAO, 1992.

Hospital Centro Oriente-HCo. Agenda local, localidad Santa Fe.Bogotá, Colombia. Octubre 2011. En: http://www. saludcapital.gov.co [consultado el 18 de abril de 2013].

Programa de las Naciones Unidas para el Desarrollo-Pnud. Reducción de la pobreza y la desigualdad. 2011. En: http://www.pnud.cl/areas/2. asp [consultado el 09 de febrero de 2013].

Secretaría de Salud, Alcaldía Mayor de Bogotá. Avance de los recorridos y diagnóstico rápido de los territorios saludable.
Bogotá, Colombia: Hospital Centro Oriente HCO, 2013.

Secretaría Distrital de Integración Social-SDIS. "Secretaría Distrital de Integración". Informe Localidad Santa Fe. Colombia, 2010. En: <http://www.integracionsocial. gov.co/> [consultado el 23 de abril de 2013].

Segovia, Adriana. Universidad Nacional de Colombia. Colombia, 2012. En: $<$ http://www.bdigital.unal.edu. co/8950/> [consultado el 27 de abril de 2013].

Fecha de ingreso: 20/09/2013

Fecha de aprobación: 04/03/2014 